\title{
Evaluación de líneas de arroz (Oryza sativa L.) y estabilidad fenotípica en cinco localidades de Río San Juan, Nicaragua
}

\section{Evaluation of rice lines (Oryza sativa L.) and phenotypic stability in five locations of Río San Juan, Nicaragua}

\author{
Yessenia Sandoval Balladares', Álvaro Benavides González², Vidal Marín Fernández ${ }^{2}$ \\ ${ }^{1}$ MSc. Instituto Nicaraguense de Tecnología Agropecuaria (INTA), ORCID: https://orcid.org/0000-0002-9330-6708, sandovalcarmen3000@ \\ yahoo.es \\ ${ }^{2}$ MSc. Docntes Universidad Nacional Agraria (UNA), ORCID: https://orcid.org/0000-0002-0596-690X, alvaro.benavides@ci.una.edu.ni
}

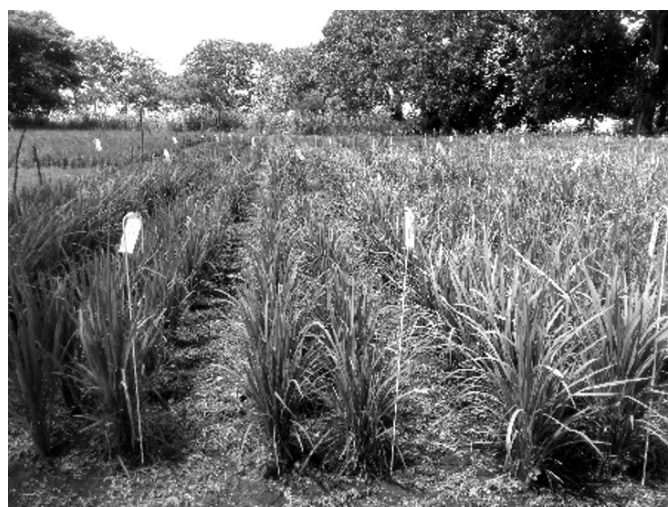

\section{RESUMEN}

Fueron estudiadas líneas de arroz (Oryza sativa L.) provenientes del CIAT (Colombia) y del Instituto Nicaragüense de Tecnología Agropecuaria (INTA) en colaboración con la Misión Técnica de China Taiwán, y estudiados en ambientes del departamento de Río San Juan (San Carlos, El Castillo, San Miguelito, Los Cerritos y Los Pantanos) durante el período 2014-2016. En diseños Alfa Látice (5x5) se evaluaron 25 materiales genéticos en San Carlos, El Castillo, San Miguelito, seleccionadas 10 líneas y organizadas en diseños de Bloques Completos al Azar (BCA) en Los Cerritos y Los Pantanos y empleados modelos univariados y mixtos en la interacción genotipo-ambiente (GxA). En las localidades de San Carlos, El Castillo y San Miguelito, sobresalieron las líneas CT18238-23-6-1-4-1-2-M (T10), CT18247-11-5-2-3-2-2-M (T14) y los testigos INTA Dorado e INTA San Juan con rendimientos superior a los $8200 \mathrm{~kg} \mathrm{ha}^{-1}$. En Los Cerritos, la línea CT18238-23-6-1-4-1-2-M (T10) superó los 11 $500 \mathrm{~kg} \mathrm{ha}^{-1}$, con buena aceptabilidad fenotípica y tolerante a $\mathrm{Hel}$ mistosporium y Pirycularia. Las metodologías utilizadas en GxA, determinaron que los genotipos T15 (PCT-4 $\backslash \mathrm{SA} \backslash 1 \backslash 1, \mathrm{BO} \backslash 3 \backslash 1>60-3$ 5-2-M) y T24 (M-57-191) fueron inestables y con bajos rendimientos. No obstante, los genotipos T6 (CT18233-15-6-6-4-1-3-M) y T7 (CT18245-11-6-2-3-4-3-M) resultaron estables y con rendimientos superiores al promedio. Los genotipos INTA San Juan e INTA Dorado, tuvieron rendimientos intermedios y altos en algunos ambientes, pero genotipos estables con otras metodologías de análisis.

Palabras clave: Alfa Látice, genotipo, interacción genotipo ambiente, material genético.
ABSTRACT
Rice lines (Oryza sativa L.) from CIAT (Colombia) and Nicaraguan Institute of Agricultural Technology (INTA) in collaboration with the Technical Mission of China Taiwan, and evaluated in environments of the San Juan River Department (San Carlos, El Castillo, San Miguelito, Los Cerritos and Los Pantanos) during the period 2014-2016. In Alpha Lattice designs $(5 \times 5)$ they tested 25 genetic materials in San Carlos, El Castillo, San Miguelito, selected 10 lines and organized in designs Complete Blocks Random (RCB) in Los Cerritos and Los Pantanos, and employees univariate models and mixed in the Genotype-environment interaction (GxE). In the towns of San Carlos, El Castillo and San Miguelito, the lines CT18238-236-1-4-1-2-M (T10), CT18247-11-5-2-3-2-2-M stood out (T14) and the witnesses INTA Dorado and INTA San Juan with yields greater than $8,200 \mathrm{~kg} \mathrm{ha}^{-1}$. In Los Cerritos, line CT18238-23-6-1-4-1-2-M (T10) exceeded $11,500 \mathrm{~kg} \mathrm{ha}^{-1}$, with good phenotypic acceptability and tolerant to Helmistosporium and Pirycularia. The methodologies used in GxE, determined that the T15 genotypes (PCT-4 $\mathrm{SA} \backslash 1 \backslash 1, \mathrm{BO} \backslash 3 \backslash 1>60-3-5-2-\mathrm{M})$ and T24 (M-57-191) were unstable and with low yields. However, the genotypes T6 (CT18233-15-6-64-1-3-M) and T7 (CT18245-11-6-2-3-4-3-M) were stable and with good performance. The genotypes INTA San Juan and INTA Dorado, had intermediate and high yields in some environments, but stable genotypes with other analysis methodologies.

Keywords: Alpha Lattice, genotype, environment genotype interaction, genetic material.
Recibido: 2 de abril del 2019

Aceptado: 30 de mayo del 2019

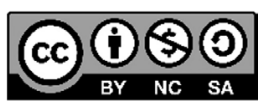

Los artículos de la revista La Calera de la Universidad Nacional Agraria, Nicaragua, se comparten bajo términos de la licencia Creative Commons: Reconocimiento, No Comercial, Compartir Igual. Las autorizaciones adicionales a las aquí delimitadas se pueden obtener en el correo edgardo.jimenez@ci.una.edu.ni

C Copyright 2019. Universidad Nacional Agraria 
$\mathrm{E}$ 1 arroz (Oryza sativa L.) es el cultivo más importante en la alimentación humana (Morejón et al. 2015), segundo entre los cereales y alimento básico para más de un tercio de la población mundial (Orona et al. 2013). Su producción promedio anual alcanza aproximadamente unos 650 millones de toneladas. A pesar de su categoría alimenticia, la producción de grano no cubre la demanda mundial (Hernández-Rodríguez et al. 2014).

El arroz es básico en países de América Central, y en Nicaragua aporta el 14\% de la canasta básica; abarca casi 85 000 hectáreas y representa el $64 \%$ del área total de producción (MAGFOR, 2005; citado por Trouchez et al. 2006). En condiciones de secano, los rendimientos son muy variables según la región y sistema de producción, además depende de las condiciones pluviométricas y fitosanitarias, variando de 2-3 t ha- ${ }^{1}$ en sistemas de secano, y de 4-6 $\mathrm{t} \mathrm{ha}^{-1}$ en sistemas mecanizados (Trouchez et al. 2006). La actividad arrocera genera 191 millones de dólares a nivel nacional, y crea alrededor de 75000 puestos de trabajo directos e indirectos al año (ANAR, 2011; citado por Téllez y Rivera, 2017).

Las variedades de arroz constituyen un componente tecnológico importante, por lo que se requiere incorporar genotipos que se adapten a diferentes condiciones ambientales y manejo agronómico sin deterioro de la productividad y sostenibilidad de agroecosistemas. El mejoramiento genético ha sido esencial en la búsqueda de genotipos que contribuyan a incrementar la productividad y calidad de los productos agrícolas.

La obtención de líneas de arroz mediante el mejoramiento participativo es una estrategia empleada en el país para elevar la producción a fin de alcanzar la seguridad, soberanía alimentaria y adaptación al cambio climático. No obstante, hay limitaciones de recursos financieros, disponibilidad de semilla, etc., por lo que es sustancial la selección en ambientes distintos (Camargo-Buitrago et al. 2011a, 2011b).

Los mejoradores asumen que la interacción genotipo-ambiente es un gran problema, y por esto realizan pruebas de estabilidad y de adaptación para identificar genotipos de interés. La detección de interacción genotipo-ambiente en ensayos de campo ha llevado al desarrollo de procedimientos de estabilidad (Abbott y Pistorale, 2011). Pérez (2014), considera que la utilización de técnicas multivariadas al estudio de las interrelaciones entre cultivares, ambientes, variables o sus interacciones, es una herramienta valiosa para la identificación de material genético sobresaliente.

El presente estudio empleó técnicas univariantes y multivariantes para evaluar líneas de arroz, y determinar estabilidad e interacción genotipo-ambiente (GxA) del rendimiento en cinco ambientes del departamento de Río San Juan, Nicaragua.

\section{MATERIALES Y MÉTODOS}

Descripción de los experimentos. El material genético inicial fue obtenido de la evaluación realizada por el centro de referencia nacional del cultivo de arroz, TAINIC en el año 2013. Los experimentos se establecieron en localidades del departamento de Río San Juan. Las actividades agronómicas fueron de manera convencional. En el Cuadro 1, se presenta información de las localidades, condiciones agroclimáticas (INETER, 2018), coordenadas (UTM, 16N), edáficas y de cosecha.

La primera etapa consistió en la evaluación de 25 genotipos de arroz en Bloques Incompletos, y en la segunda etapa se evaluaron los mejores 10 materiales de arroz en Bloques Completos al Azar mediante Pruebas Avanzadas de Rendimiento (PAR).

Material biológico. En el Cuadro 2, muestra los genotipos provenientes de la selección en vivero de 100 líneas mejoradas avanzadas (F6) provenientes de cruces convencionales (hibrido simple) e interespecíficos proveídas por el CIAT. Posterior a la primera etapa de evaluación se seleccionaron 10 líneas, las que pasaron a evaluarse a través de Pruebas Avanzadas de Rendimiento (PAR).

Cuadro 1. Información sobre los ambientes y momentos de cosecha

\begin{tabular}{lccccccccc}
\hline Localidad & $\mathrm{T}$ & $\mathrm{HR}$ & Prec. & Ins. & Este & Norte & Suelo & Siembra & Cosecha \\
\hline San Carlos* $^{*}$ & 28.8 & 90.2 & 549.9 & 110.7 & 761701 & 1226243 & Arcilloso & jun-14 & nov-14 \\
El Castillo* $^{*}$ & 31.8 & 89.9 & 876.6 & 110.0 & 790209 & 1242398 & Arcilloso & jun-15 & nov-15 \\
San Miguelito* $^{*}$ & 31.2 & 90.1 & 575.1 & 149.7 & 741660 & 1260852 & Franco-Arcilloso & jun-15 & nov-15 \\
Los cerritos (Morrito) $^{* *}$ & 30.7 & 91.5 & 675.8 & 129.0 & 722629 & 1291858 & Franco-Arcilloso & jul-16 & dic-16 \\
San Miguelito (Los Pantanos) $^{* *}$ & 27.2 & 88.5 & 990.0 & 129.3 & 731961 & 1265715 & Franco-Arcilloso & jul-16 & dic-16 \\
\hline
\end{tabular}

*=Primera etapa, ${ }^{* *}=$ Segunda etapa. $\mathrm{T}=$ Temperatura $\left({ }^{\circ} \mathrm{C}\right), \mathrm{HR}=$ Humedad Relativa (\%), Prec=Precipitación Acumulada (mm/año), Ins.=Insolación Acumulada (Horas sol/año). 
Cuadro 2. Descripción del material biológico de arroz estudiado

\begin{tabular}{|c|c|c|c|}
\hline Tratamientos & Código del genotipo & Tratamientos & Código del genotipo \\
\hline $\mathrm{T} 1$ & CT18667-5-17-3-4-3-M & $\mathrm{T} 14$ & CT18247-11-5-2-3-12-2M \\
\hline $\mathrm{T} 2$ & CT18614-4-1-2-3-1-M & $\mathrm{T} 15$ & PCT-4\SA $\backslash 1 \backslash 1, \mathrm{BO} \backslash 3 \backslash 1>60-3-5-2-\mathrm{M}$ \\
\hline T3 & CT18620-6-5-5-2-3-M & $\mathrm{T} 16$ & PCT- $11 \backslash 0 \backslash 0 \backslash 2, \mathrm{BO} \backslash 2 \backslash 1>60-3-1-1-\mathrm{M}$ \\
\hline $\mathrm{T} 4$ & FLO3724-3P-5-1P-3P-M & $\mathrm{T} 17$ & PCT- $11 \backslash 0 \backslash 0 \backslash 2, \mathrm{BO} \backslash 2 \backslash 1>46-\mathrm{M}-4-4-2-3-\mathrm{M}$ \\
\hline T5 & PCT-11 $\backslash 0 \backslash 0 \backslash 2, \mathrm{BO} \backslash 2-1>19-\mathrm{M}-3-4$ & $\mathrm{~T} 18$ & PCT-4\SA $\backslash 1 \backslash 1, \mathrm{BO} \backslash 3 \backslash 1>60-3-2-1-\mathrm{M}$ \\
\hline T6 & CT18233-15-6-6-4-1-3-M & $\mathrm{T} 19$ & PCT- $11 \backslash 0 \backslash 0 \backslash 2, \mathrm{BO} \backslash 2 \backslash 1>46-\mathrm{M}-4-2-1-5-\mathrm{M}$ \\
\hline $\mathrm{T} 7$ & CT18245-11-6-2-3-4-3-M & $\mathrm{T} 20$ & INTA Dorado (Testigo) \\
\hline $\mathrm{T} 8$ & CT18238-23-1-1-2-5-1-M & $\mathrm{T} 21$ & PCT- $11 \backslash 0 \backslash 0 \backslash 2, \mathrm{BO} \backslash 2 \backslash 1>92-\mathrm{M}-2-1-4-5-\mathrm{M}$ \\
\hline T9 & CT18238-23-1-2-3-3-1-M & $\mathrm{T} 22$ & CT17334-13-7-2-1-1-4-3-1-M \\
\hline $\mathrm{T} 10$ & CT18238-23-6-1-4-1-2-M & $\mathrm{T} 23$ & CT17130-M-1-2-1-1-2-2-4-M \\
\hline T11 & PCT- $11 \backslash 0 \backslash 0 \backslash 2, \mathrm{BO} \backslash 2 \backslash 1>19-\mathrm{M}-3-2$ & $\mathrm{~T} 24$ & M-57-191 \\
\hline $\mathrm{T} 12$ & CT18245-18-2-4-1-2-2-M & $\mathrm{T} 25$ & INTA San Juan (Testigo) \\
\hline $\mathrm{T} 13$ & CT18247-11-5-2-3-1-1-M & & \\
\hline
\end{tabular}

plantas de la parcela útil. La información manejada en hojas electrónicas fue procesada con GEA-R (Pacheco et al. 2015) del CIMMYT, SAS (SAS Institute, 2002) e InfoGen (Balzarini, 2005) Análisis paramétrico
univariantes y multi-
variantes. Durante la
primera etapa se es-
tablecieron modelos
mixtos en las varia-
bles provenientes de
ensayos de Bloques
Incompletos (Alfa
Látice), y en la segunda etapa los análisis estadísticos correspondieron a diseños de Bloques Completos al Azar. Al conjunto de datos provenientes de los cinco ambientes se les aplicaron modelos univariantes (Russell, Shuckla y Wricke's) y multivariantes (AMMI, SREG, PLS).

El análisis de regresión conjunta corroboró el comportamiento de genotipos en los diferentes ambientes según Eberhart y Russell (1966); citado por Williams (2010) y González (2001). La metodología de Suckla (1972); citado por González et al. (2010); y Ecovalencia ( $\mathrm{W}_{\mathrm{i}}$ ) de Wricke (1962); citado por González (2001) fue empleada con el mismo propósito. La interacción fue estudiada mediante el Análisis de Componentes Principales (ACP) de Efectos Aditivos e Interacción Multiplicativa (AMMI) de Gauch y Zobel (1988); citados por Samonte et al. (2005); Balzarini et al. (2005); el modelo de regresión por sitio (SREG) sugeridos por Samonte et al. (2005), Balzarini et al. (2005), Sánchez et al. (2014) y Lozano et al. (2015). El método Factorial Regresión o PLS (Partial Least Square) desarrollado por Wold (1966); citado por Márquez (2017), para determinar interacción y relación de variables externas con genotipos y ambientes (Pacheco et al. 2015).

\section{RESULTADOS Y DISCUSIÓN}

Relación de 25 genotipos en tres ambientes de Río San Juan. Las variables mostraron significación estadística en los factores estudiados, a excepción de la repetición. En cuanto al rendimiento, la mayor varianza en el modelo la proporcionó las repeticiones y genotipos. La interacción GxA altamente significativa $(\mathrm{Pr}=0.0001)$ indicó correspondencia entre los factores estudiados (Cuadro 3). 
Cuadro 3. Desviación de los cuadrados medios en factores y significación de las variables evaluadas en 25 genotipos de arroz (Oryza sativa L.) durante el ciclo de primera 2014-2015. San Carlos, El Castillo y San Miguelito, Río San Juan

\begin{tabular}{lrrrrrrr}
\hline Variables & \multicolumn{1}{c}{$\begin{array}{c}\mathrm{A} \\
\mathrm{gl}=2\end{array}$} & \multicolumn{1}{c}{$\begin{array}{c}\mathrm{R} \\
\mathrm{gl}=2\end{array}$} & $\begin{array}{c}\mathrm{B}(\mathrm{R}) \\
\mathrm{gl}=12\end{array}$ & \multicolumn{1}{c}{$\begin{array}{c}\mathrm{G} \\
\mathrm{gl}=24\end{array}$} & \multicolumn{1}{c}{$\begin{array}{c}\mathrm{G} * \mathrm{~A} \\
\mathrm{gl}=48\end{array}$} & $\mathrm{R}^{2}$ & $\mathrm{CV}$ \\
\hline Rendimiento & $1381.93^{\mathrm{NS}}$ & $4558.29^{\mathrm{NS}}$ & $2427.50^{* *}$ & $3394.54^{* *}$ & $1938.01^{* *}$ & 0.79 & 23.1 \\
Macollamiento & $14.35^{\mathrm{NS}}$ & $1.07^{\mathrm{NS}}$ & $4.17^{* *}$ & $5.66^{* *}$ & $2.63^{\mathrm{NS}}$ & 0.72 & 11.7 \\
Altura del tallo & $39.33^{* *}$ & $5.15^{\mathrm{NS}}$ & $7.20^{* *}$ & $3.43^{* *}$ & $1.99^{* *}$ & 0.85 & 4.1 \\
Floración & $9.85^{* *}$ & $1.87^{\mathrm{NS}}$ & $1.34^{*}$ & $17.78^{* *}$ & $1.06^{\mathrm{NS}}$ & 0.98 & 1.2 \\
Madurez & $6.33^{*}$ & $0.75^{\mathrm{NS}}$ & $2.07^{\mathrm{NS}}$ & $16.54^{* *}$ & $5.15^{* *}$ & 0.96 & 1.6 \\
\hline
\end{tabular}

$\mathrm{A}=$ Ambiente, $\mathrm{G}=$ Genotipo, $\mathrm{R}=$ Repetición, $\mathrm{B}=\mathrm{Bloque}, \mathrm{gl}=$ Grados de libertad, $\mathrm{R}^{2}=$ Coeficiente de determinación,

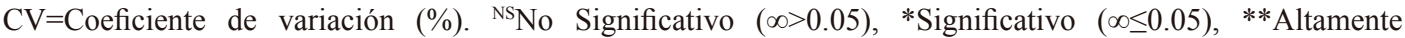
Significativo $(\infty \leq 0.01)$.

La variación fue considerable en los factores y la eficiencia del modelo en Látice superó en muchos casos al modelo en BCA. El bloqueo a lo interno de las repeticiones, así como los genotipos mostraron efecto altamente significativo $(\operatorname{Pr}<0.01)$ en variables de crecimiento, desarrollo y rendimiento (Cuadro 4).

Según Doyle (1986); citado por Cuadra (2016), los factores más importantes que afectan al rendimiento del cultivo del arroz son el potencial de la variedad y la reacción a la fertilización, manejo de plagas, riego y condiciones ambientales. tabilidad fenotípica propuesta por los productores de arroz de Río San Juan.

El genotipo T10 mostró el mayor rendimiento promedio (9 $\left.436.5 \mathrm{~kg} \mathrm{ha}^{-1}\right)$, y estadísticamente superó a los otros genotipos. Los genotipos T6, T14 e INTA Dorado puntearon los $8000 \mathrm{~kg} \mathrm{ha}^{-1}$. El mejor promedio ambiental se consiguió en Los Cerritos (9 $343.3 \mathrm{~kg} \mathrm{ha}^{-1}$ ). Los genotipos T4 y T5 revelaron una menor variación en cuanto a los rendimientos logrados, T15 e INTA Dorado manifestaron una mayor desviación en los ambientes (Cuadro 5).

Cuadro 4. Desviación de los cuadrados medios en factores y significación de las variables evaluadas en 25 genotipos de arroz (Oryza sativa L.) durante el ciclo de primera 2014-2015, San Carlos, Río San Juan

\begin{tabular}{|c|c|c|c|c|c|c|}
\hline Variables & $\begin{array}{c}\mathrm{R} \\
\mathrm{gl}=2\end{array}$ & $\begin{array}{l}\mathrm{B}(\mathrm{R}) \\
\mathrm{gl}=12\end{array}$ & $\begin{array}{c}\mathrm{G} \\
\mathrm{gl}=24\end{array}$ & $\mathrm{R}^{2}$ & $\begin{array}{l}\text { CV } \\
(\%)\end{array}$ & $\begin{array}{l}\text { ER } \\
(\%)\end{array}$ \\
\hline \multicolumn{7}{|l|}{ San Carlos } \\
\hline Rendimiento & $3982.71 *$ & $1910.54 *$ & $2307.50 * *$ & 0.77 & 19.83 & 101.31 \\
\hline Macollamiento & $3.93^{\mathrm{NS}}$ & $2.79 *$ & $2.86^{* *}$ & 0.71 & 9.10 & 97.31 \\
\hline Altura del tallo & $2.02^{\mathrm{NS}}$ & $9.79 * *$ & $12.67 * *$ & 0.97 & 1.61 & 101.36 \\
\hline Floración & $1.62^{\mathrm{NS}}$ & $6.81 * *$ & $10.33^{* *}$ & 0.99 & 0.77 & 126.12 \\
\hline Madurez & $0.92^{\mathrm{NS}}$ & $5.02 * *$ & $7.69 * *$ & 0.99 & 0.45 & 120.96 \\
\hline \multicolumn{7}{|l|}{ El Castillo } \\
\hline Rendimiento & $5901.78 * *$ & $2297.16^{* *}$ & $2541.75 * *$ & 0.79 & 23.06 & 109.77 \\
\hline Macollamiento & $4.74^{\mathrm{NS}}$ & $3.46^{* *}$ & $3.20 *$ & 0.69 & 10.57 & 105.69 \\
\hline Altura del tallo & $0.23^{\mathrm{NS}}$ & $10.53 * *$ & $13.64 * *$ & 0.99 & 0.99 & 100.11 \\
\hline Floración & $0.03^{\mathrm{NS}}$ & $6.67 * *$ & $10.54 * *$ & 0.99 & 0.52 & 117.30 \\
\hline Madurez & $2.58^{\mathrm{NS}}$ & $5.21 * *$ & $7.12 * *$ & 0.91 & 1.82 & 96.74 \\
\hline \multicolumn{7}{|l|}{ San Miguelito } \\
\hline Rendimiento & $3160.22^{\mathrm{NS}}$ & $2468.68 * *$ & $2592.06^{* *}$ & 0.74 & 23.61 & 115.23 \\
\hline Macollamiento & $7.88^{\mathrm{NS}}$ & $4.26 * *$ & $4.93 * *$ & 0.80 & 13.40 & 134.78 \\
\hline Altura del tallo & $6.70^{\mathrm{NS}}$ & $15.01 *$ & $9.77 * *$ & 0.74 & 6.21 & 93.30 \\
\hline Floración & $1.61^{\mathrm{NS}}$ & $7.92 * *$ & $10.03 * *$ & 0.97 & 1.85 & 100.01 \\
\hline Madurez & $2.31^{\mathrm{NS}}$ & $11.31 * *$ & $14.33^{* *}$ & 0.97 & 2.03 & 100.05 \\
\hline
\end{tabular}

$\mathrm{R}=$ Repetición, $\mathrm{B}=\mathrm{Bloque}, \mathrm{G}=$ Genotipo, $\mathrm{gl}=$ Grados de libertad, $\mathrm{R}^{2}=$ Coeficiente de determinación, $\mathrm{CV}=$ Coeficiente de variación, ER=Eficiencia Relativa. ${ }^{\text {NS}}$ No Significativo $(\infty>0.05), *$ Significativo $(\infty \leq 0.05), * *$ Altamente Significativo $(\infty \leq 0.01)$. 
Cuadro 5. Rendimientos promedios de 10 genotipos de arroz (Oryza sativa L.) evaluados en cinco ambientes. Río San Juan

\begin{tabular}{|c|c|c|c|c|c|c|c|}
\hline Tratamientos & San Carlos & El Castillo & San Miguelito & Los Cerritos & Los Pantanos & Media & DE \\
\hline $\mathrm{T} 10$ & 9024.9 & 8883.0 & 8966.7 & 11539.7 & 8066.9 & $9436.5 \mathrm{a}$ & 1313.1 \\
\hline T6 & 7999.6 & 6813.1 & 7720.4 & 10316.1 & 7166.6 & $8147.7 \mathrm{~b}$ & 1373.5 \\
\hline $\mathrm{T} 14$ & 8390.8 & 8513.9 & 7520.8 & 9132.4 & 6244.7 & $8033.8 \mathrm{~b}$ & 1118.3 \\
\hline INTADor & 8763.3 & 8853.3 & 5954.4 & 10009.7 & 5858.3 & $8020.4 \mathrm{~b}$ & 1874.7 \\
\hline $\mathrm{T} 7$ & 6445.4 & 7663.1 & 7213.7 & 9925.6 & 7344.5 & $7950.5 \mathrm{~b}$ & 1312.4 \\
\hline INTASJ & 8200.9 & 8032.2 & 6042.9 & 10081.5 & 6196.9 & $7859.1 \mathrm{~b}$ & 1661.1 \\
\hline T5 & 7534.8 & 7419.4 & 6693.2 & 7924.2 & 7866.8 & $7515.0 \mathrm{bc}$ & 493.0 \\
\hline $\mathrm{T} 4$ & 7599.8 & 8028.2 & 6184.8 & 7059.5 & 6010.4 & $6981.7 \mathrm{bc}$ & 874.9 \\
\hline $\mathrm{T} 15$ & 5196.7 & 5293.9 & 4849.2 & 9389.4 & 7184.9 & $6570.8 \mathrm{~cd}$ & 1912.3 \\
\hline $\mathrm{T} 24$ & 4845.5 & 4862.9 & 6395.9 & 8054.8 & 6330.9 & $6220.3 \mathrm{~d}$ & 1329.1 \\
\hline Media & $7443.3 \mathrm{~b}$ & $7436.3 \mathrm{~b}$ & $6754.2 \mathrm{~b}$ & $9343.3 \mathrm{a}$ & $6827.1 \mathrm{~b}$ & & \\
\hline $\mathrm{DE}$ & 1446.5 & 1399.2 & 1147.9 & 1334.8 & 797.0 & & \\
\hline $\mathrm{R}$ & 0.2056 & 0.0855 & 0.3482 & 0.0620 & 0.2639 & & \\
\hline A & & & & & & 0.0001 & \\
\hline G & 0.0013 & 0.0247 & 0.3803 & 0.0092 & 0.0001 & 0.0006 & \\
\hline GxA & & & & & & 0.0261 & \\
\hline $\mathrm{R}^{2}$ & 0.76 & 0.64 & 0.51 & 0.64 & 0.93 & 0.7238 & \\
\hline $\mathrm{CV}$ & 14.26 & 19.08 & 27.94 & 14.33 & 4.03 & 17.211 & \\
\hline
\end{tabular}

$\mathrm{R}=$ Repetición, $\mathrm{A} .=$ Ambiente, $\mathrm{G}=$ Genotipo, $\mathrm{CV}=$ Coeficiente de Variación (\%). $\mathrm{R}^{2}=$ Coeficiente de Determinacción, Medias con letras en común son iguales (Tukey, $\infty>0.05$ ), DE=Desviación Estándar.

Los resultados obtenidos de las diferentes evaluaciones fueron sujetos a metodologías univariantes y multivariantes para determinar estabilidad e interacción del genotipo con el ambiente. Samonte et al. (2005), Wade et al. (1999), Acevedo et al. (2010), González et al. (2010), Díaz et al. (2015), Díaz et al. (2017) y Anyaoha et al. (2018), hacen mención sobre la aplicación de estas metodologías.

\section{Métodos univariantes}

Modelo de regresión conjunta. El análisis de estabilidad de Eberhart y Russell a partir de los índices ambientales, aglutinó como estables a los genotipos T6 (8 $\left.003.14 \mathrm{~kg} \mathrm{ha}^{-1}\right)$ y T7 (7 $\left.718.45 \mathrm{~kg} \mathrm{ha}^{-1}\right)$. INTA San Juan, INTA Dorado y T14 superaron al promedio general (IC=7 552 \pm 914 ), pero estuvieron fuera del límite de estabilidad (Figura 1). También se puede interpretar que los genotipos T10, T6, T7 y T14, mostraron buena respuesta en todos los ambientes y fueron predecibles $\left(b_{i}=1 ; S^{2} d i=0\right)$. Dentro del conjunto con mejor respuesta en ambientes desfavorable y predecibles $\left(b_{i}<1 ; S^{2} d i=0\right)$ sobresalió el genotipo T5, esta aseveración coincide con AMMI y SREG (Figura 3).
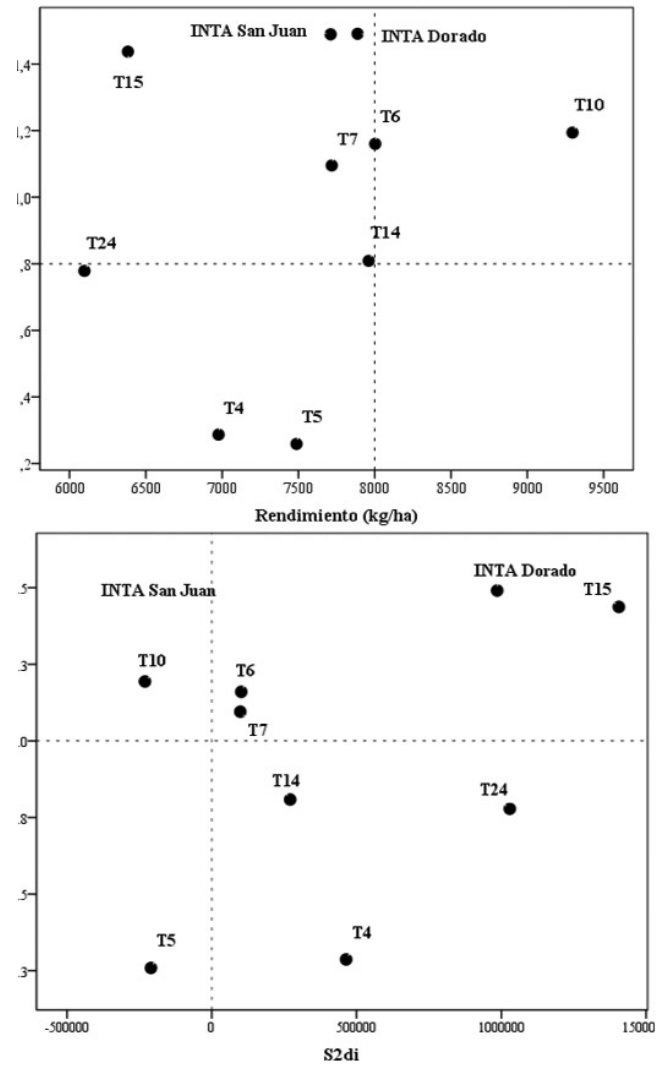

Figura 1. Dispersión bidimensional del coeficiente de regresión $\left(\mathrm{b}_{\mathrm{i}}\right)$ en función del rendimiento $\left(\mathrm{kg} \mathrm{ha}^{-1}\right)$ promedio de diez genotipos de arroz y bi en función de la desviación ( $\mathrm{S}^{2} \mathrm{di}$ ) evaluadas en el departamento de Río San Juan, 2014-2016. 
Abbott y Pistorale (2011), plantean un modelo de regresión lineal para estudiar estabilidad y adaptabilidad fenotípica en cultivares. El coeficiente $b_{i}$ mide la respuesta a la variación ambiental, mientras que la varianza de la desviación de variabilidad ( $\left.\mathrm{S}^{2} \mathrm{di}\right)$ es una medida de estabilidad (González, 2001; Cadena-Guerrero et al. 2019). Este parámetro no es suficiente, y la media total de cada genotipo debería considerarse; así, los genotipos con pendiente cercana a uno y alto rendimiento serán bien adaptados a los ambientes (Finlay y Wilkinson, 1963; 1966; citados por González, 2001).

Análisis de Shuckla y Wricke's (Wi). Los genotipos T6, T7, $\mathrm{T} 14$ obtuvieron valores superiores al rendimiento promedio, y catalogados como los más estables en las metodologías de Shuckla y Wricke's (Figura 2). Estas aseveraciones contrastan con resultados de Eberhart y Russell, lo que indicó efectividad en los métodos univariantes para estudiar estabilidad de genotipos de arroz en los ambientes.
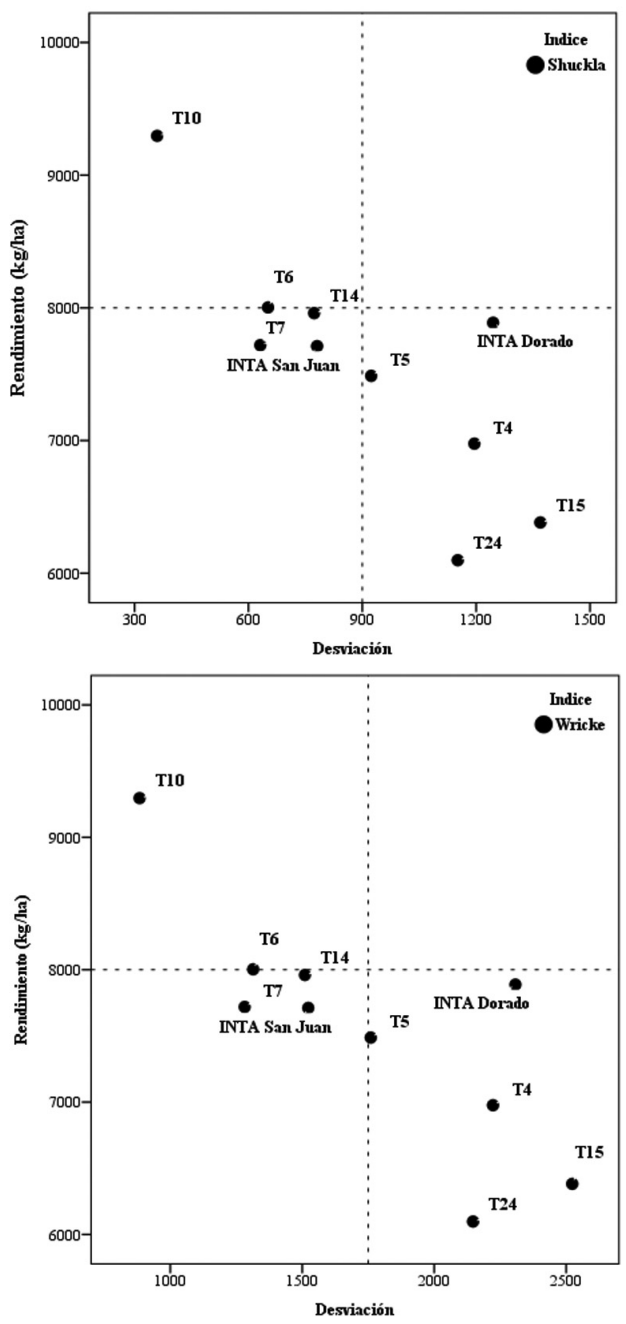

Figura 2. Dispersión bidimensional del coeficiente de Shuckla y Wricke's en función del rendimiento $\left(\mathrm{kg} \mathrm{ha}^{-1}\right)$ promedio de diez genotipos de arroz evaluadas en el departamento de Río San Juan, 2014-2016.
El coeficiente $\mathrm{W}_{\mathrm{i}}$ mide la contribución del genotipo a la interacción GxA, y los genotipos con $\mathrm{W}_{\mathrm{i}}$ próximos a cero son más estables (González, 2001; Abbott y Pistorale, 2011). La suma de residuos de cada genotipo en todos los ambientes precisa un ANDEVA como modelo aditivo lineal (Wricke, 1962; citado por González, 2001). Castañón (1994), menciona el uso de cualquier método para estudios de parámetros de estabilidad de líneas avanzadas y variedades de arroz, y señala a Wi como el método más sencillo e interpretación fácil en comparación a Eberhart y Russell.

\section{Métodos multivariantes}

Método AMMI. En el ANDEVA combinado del Cuadro 6, se aprecian diferencias altamente significativas entre ambientes, genotipos $(\mathrm{P}<0.001)$ y efectos significativos en la interacción GxA ( $\mathrm{Pr}=0.0261)$. Los genotipos se comportaron de manera heterogénea a la variación ambiental. El modelo AMMI determinó una amplia variación en el ambiente $(41.79 \%$ ) y genotipos (31.06\%). También comprobó el aporte a la variación total de la interacción GxA (27.15 \%). La prueba de Gollob reveló que el PC-1 fue altamente significativo ( $\mathrm{Pr}=0.0005), \mathrm{y}$ el PC-2 aportó $25.46 \%$ de la variación, pero no fue significativo $(\mathrm{Pr}=0.0851)$ al $95 \%$ de confianza. En la metodología AMMI el PC-1 (58.33\%) y PC-2 (25.46\%) explicaron 83.8 $\%$ de la variación relacionada a GxA. Los gráficos biplot expresaron muy bien el modelo de dependencia (Figura 3).

González (2001) y Pérez et al. (2014), sugieren que un $75 \%$ aislado en los PC-1 y PC-2, es deseable para interpretar confiablemente las correlaciones aproximadas en el biplot.

Cuadro 6. Análisis de varianza del rendimiento mediante el modelo AMMI y SREG en 10 genotipos de arroz (Oryza sativa L.) evaluados en cinco ambientes. Río San Juan

\begin{tabular}{|c|c|c|c|c|c|c|c|}
\hline \multirow[b]{2}{*}{ Fuente } & \multirow[b]{2}{*}{$\mathrm{gl}$} & \multicolumn{3}{|c|}{ AMMI } & \multicolumn{3}{|c|}{ SREG } \\
\hline & & Ind. & Acum. & $\operatorname{Pr}>\mathrm{F}$ & Ind. & Acum. & $\mathrm{Pr}>\mathrm{F}$ \\
\hline $\mathrm{Amb}(\mathrm{A})$ & 4 & 41.791 & 41.791 & 0.0001 & 41.7912 & 41.7912 & 0.0001 \\
\hline Gen $(G)$ & 9 & 31.060 & 72.852 & 0.0006 & 31.0603 & 72.8516 & 0.0006 \\
\hline GxA & 36 & 27.148 & 100.000 & 0.0261 & 27.1484 & 100.00 & 0.0261 \\
\hline PC-1 & 12 & 58.331 & 58.331 & 0.0005 & 62.2197 & 62.2197 & 0.0001 \\
\hline PC-2 & 10 & 25.457 & 83.789 & 0.0851 & 21.5252 & 83.7448 & 0.0018 \\
\hline PC-3 & 8 & 12.192 & 95.980 & 0.4177 & 10.5729 & 94.3177 & 0.0688 \\
\hline PC-4 & 6 & 3.992 & 99.972 & 0.8436 & 3.8241 & 98.142 & 0.4893 \\
\hline PC-5 & 4 & 0.028 & 100.00 & 0.9999 & 1.858 & 100.00 & 0.6179 \\
\hline Residual & 109 & & & & & & \\
\hline
\end{tabular}

$\mathrm{gl}=$ Grados de Libertad, Ind=Varianza Individual, Acum=Varianza Acumulada. AMMI=Efectos Aditivos e Interacción Multiplicativa, SREG=Regresión por Sitio.

El biplot de la Figura 3 (AMMI) reflejó que los genotipos T7, T5 y T4 afines a Los Pantanos, estuvieron más cercano al origen, lo que expresó mayor estabilidad, esto también coincide con la Figura 3 (SREG) del PC-1. El ge- 
notipo T10 mostró alto rendimiento y aportó a la interacción. Los genotipos T15 y T24 ubicado en la porción negativa sobre el eje de las abscisas del biplot experimentaron baja adaptación a los ambientes. El material T24 contribuyó menos a la variación del CP-1, además fueron inestables. INTA San Juan (7 $859.1 \mathrm{~kg} \mathrm{ha}^{-1}$ ) e INTA Dorado (8 $020.4 \mathrm{~kg} \mathrm{ha}^{-1}$ ), aportaron de manera significativa a la interacción GxA, pero con rendimientos discrepantes en los ambientes (Figura 3).
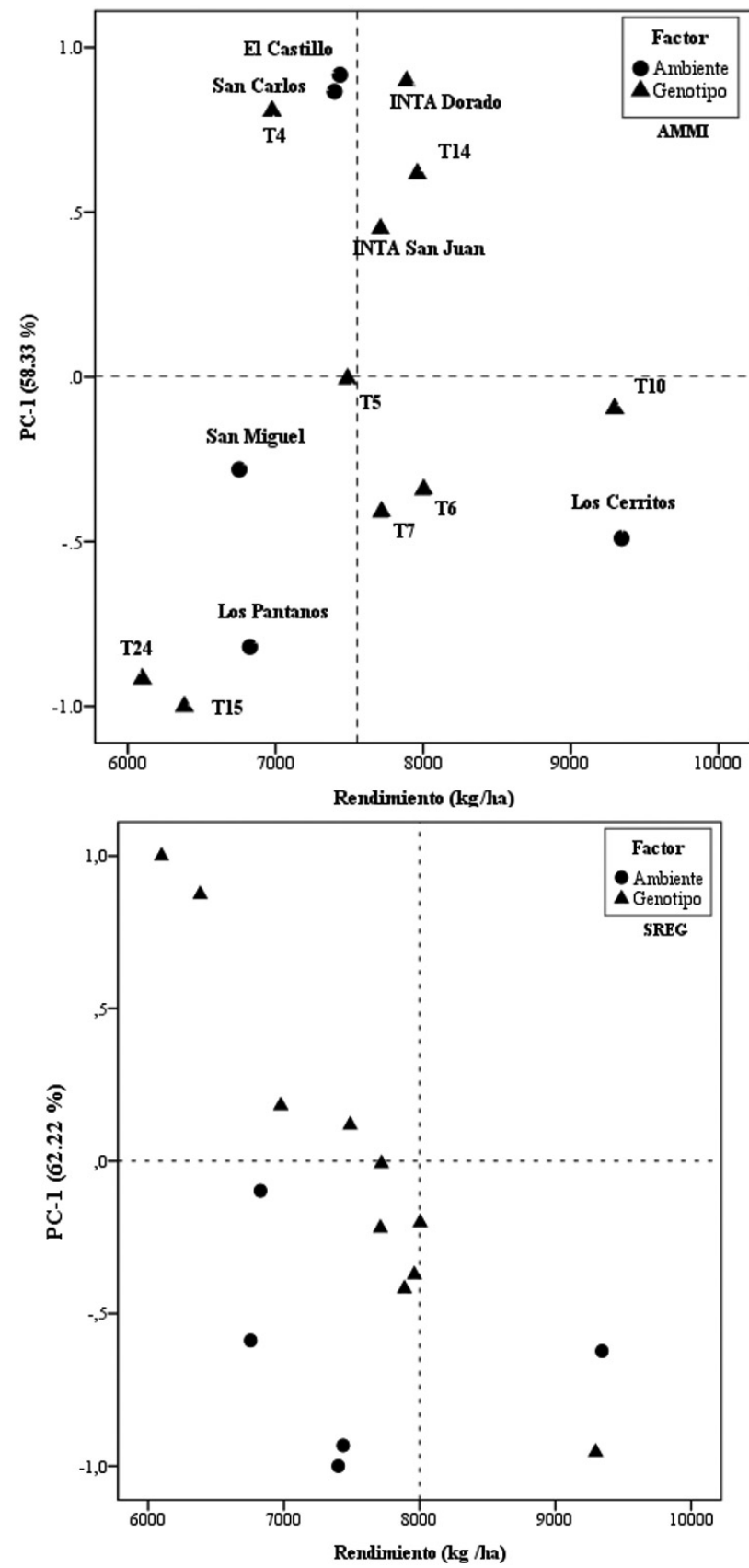

Figura 3. Dispersión bidimensional del PC-1 en función del rendimiento promedio de diez genotipos de arroz $(\mathbf{\Delta})$ en cinco condiciones ambientales ( ) del departamento de Río San Juan, 2014-2016.
Yan et al. (2000); citado por Williams et al. (2010), suponen que genotipos ubicados en sectores donde no hay ambientes son pobres en rendimiento en la mayoría de los ambientes evaluados. No obstante, AMMI no mide la estabilidad, sino el grado de interacción del genotipo con el ambiente. Cuando un genotipo tiene un valor próximo a cero, la interacción es pequeña; cuando ambos valores del ACP tienen el mismo signo, su interacción es positiva, y si son diferentes es negativa (Crossa, 2002). Samonte et al. (2005), señalan que los ambientes con valores de $\mathrm{PC}-1$ próximos a cero tienen poca interacción y baja discriminación de genotipos. Parga et al. (2011), afirman que los genotipos o ambientes con grandes valores en el ACP $(+$ ó -$)$ poseen interacciones altas, y aquellos con valores cercanos a cero, interacciones pequeñas y estables.

Método de regresión por sitios (SREG). El ANDEVA detectó diferencias altamente significaticas $(\operatorname{Pr}=0.0001)$ en los efectos principales e interacción $(\mathrm{Pr}=0.0261)$. La prueba de Gollob, asumió el $83.74 \%$ de la variación total a través de los CP-1 y CP-2, distribuida en $62.22 \%(\mathrm{PC}-1, \mathrm{Pr}=0.0001)$ y $21.52 \%$ (PC-2, $\mathrm{Pr}=0.0018)$. Los ambientes con mayor aporte a la interacción correspondieron a El Castillo y San Carlos; por el contrario, Los Pantanos mostró relación con el resto de ambientes (Figura 4). Los genotipos con estabilidad y buen rendimiento (superior al rendimiento promedio) fueron INTA San Juan y T5, contiguo a este grupo estuvo el tratamiento T14 e INTA Dorado (Figura 3).

La gráfica biplot y SREG permiten determinar el cultivar con mejor comportamiento en un ambiente específico, identifica el ambiente más apropiado, compara cultivares en un ambiente e identifica el mejor cultivar, además de diferenciar mega ambientes (Ibañéz, 2006; Sánchez et al. 2014).

Método de regresión de mínimos cuadrados parciales $(\boldsymbol{P L S})$. En el presente estudio, los años y localidades fueron estimados como ambientes, ya que las diferencias pudieron ser causa de las variaciones edafoclimáticas en cada una de las fases fenológicas del cultivo del arroz. La localidad de Los Cerritos manifestó la mayor humedad relativa promedio, y estuvo relacionada al genotipo T24. Las precipitaciones fueron mayores en Los Pantanos, El Castillo y San Carlos, y destacaron los genotipos T7, T4, T6, INTA San Juan e INTA Dorado. Los genotipos T15, T10, T14 y T5 correspondieron a la insolación de San Miguelito (Figura 4).

Abbott y Pistorale (2011), mencionan la interacción GxA en la respuesta diferencial de los genotipos evaluados en los diferentes ambientes. El método de PLS incorpora variables externas (ambientales o genotípicas) y permite estimar variables externas responsables de la variación (Pacheco et al. 2015), Cepeda y Salgueiro (2004). Sin embargo; Marquéz (2017), argumenta que el PLS no es un método, sino un conjunto de métodos con sus algoritmos asociados. 


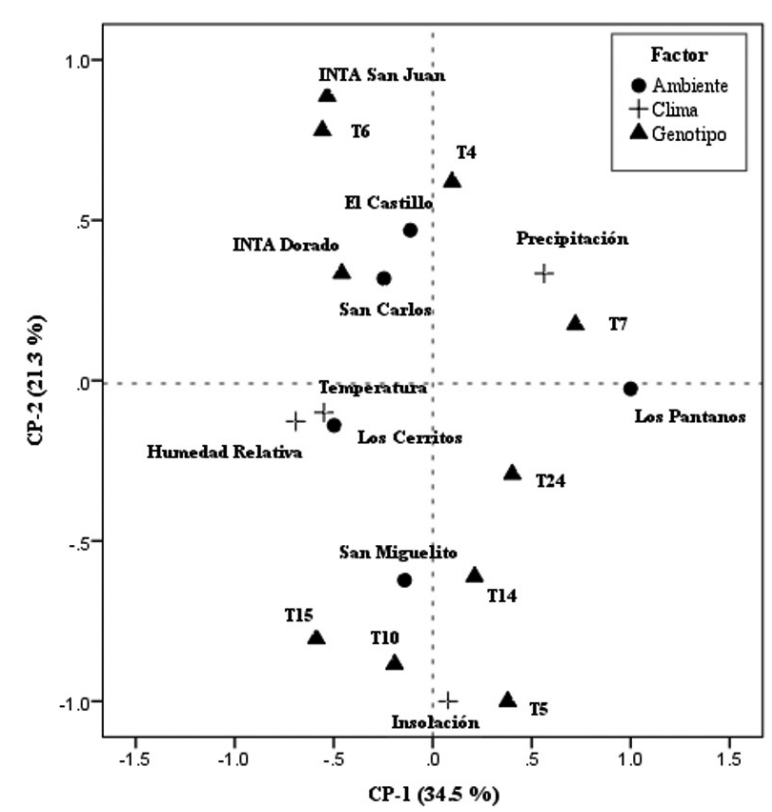

Figura 4. Dispersión bidimensional del PC-1 y PC-2 en función de variables climáticas $(+)$ de diez genotipos de arroz $(\boldsymbol{\Delta})$ en cinco condiciones ambientales ( ) del departamento de Río San Juan, 2014-2016.

\section{CONCLUSIONES}

Los resultados revelaron evidencias del comportamiento variante en los genotipos de arroz estudiados. Los genotipos T6, T7, T10 y T14 basado en los modelos de Eberhart y Russel, Shuckla y Wricke's respondieron bien en la mayoría de los ambientes, por lo que son calificados como estables y de alto rendimiento. Los testigos INTA Dorado e INTA San Juan, tuvieron un buen potencial de rendimiento, pero discrepantes en los ambientes. Las metodologías AMMI y SREG, coincidieron en que los genotipos T15 y T24 fueron inestables, de alta interacción y bajos rendimientos en los ambientes. Asimismo, el modelo AMMI precisó que los genotipos T5 (asociado a San Miguelito) y T10 (asociado a Los Cerritos) aportaron menos a la interacción; comportamiento similar tuvieron los genotipos T4, T5 y T7 (asociado a Los Pantanos). Mediante SREG, las variables de clima relacionaron a los ambientes y genotipos.

\section{AGRADECIMIENTOS}

Los autores expresan sus agradecimientos, a la misión China Taiwan, productores y promotores que participaron activamente en las actividades agrícolas de los ensayos, así como en el apoyo para el levantamiento de la información en campo.

\section{REFERENCIAS BIBLIOGRÁFICAS}

Abbott, L. y Pistorale, S. (2011). Análisis de la estabilidad y adaptabilidad de caracteres de interés agronómico en genotipos selectos de cebadilla criolla (Bromus catharticus). agriscientia, 28(2).

Acevedo, M., Reyes, E., Castrillo, W., Torres, O., Marín, C., Álvarez, R., ... y Torres, E. (2010). Estabilidad fenotípica de arroz de riego en Venezuela utilizando los modelos LIN-BINNS y AMMI. Agronomía tropical, 60(2), 131-138.

Anyaoha, C., Adegbehingbe, F., Uba, U., Popoola, B., Gracen, V., Mande, S., ... y Fofana, M. (2018). Genetic Diversity of Selected Upland Rice Genotypes (Oryza sativa L.) for Grain Yield and Related Traits. International Journal of Plant \& Soil Science, 1-9.

Balzarini, M.; C. Bruno y A. Arroyo. 2005. Análisis de ensayos agrícolas multi-ambientales. Ejemplos con Info-Gen. Ed. Brujas, Córdoba, Arg. 141 p.

Balzarini M.G., González L., Tablada M., Casanoves F., Di Rienzo J.A., Robledo C.W. (2008). Infostat. Manual del Usuario, Editorial Brujas, Córdoba, Argentina.

Cadena-Guerrero, M. M., García-Dávila, M. A., y Castro, E. (2019). Estabilidad fenotípica de genotipos de Lolium sp. en el trópico alto de Nariño, Colombia. Agronomía Mesoamericana, 483-495.

Camargo-Buitrago, I., Intire, E. M. y Górdon-Mendoza, R. (2011a). Identificación de mega-ambientes para potenciar el uso de genotipos superiores de arroz en Panamá. Pesquisa Agropecuaria Brasileira, 46(9), 1601-1069.

Camargo-Buitrago, I., Intire, Q. M. y Gordón-Mendoza, R. (2011b). Identificación de ambientes representativos y discriminatorios para seleccionar genotipos de arroz mediante el Biplot GGE. Agronomía Mesoamericana, 22(2), 245-255.

Castañón G. (1994). Estudio de la estabilidad en líneas avanzadas y variedades de arroz (Oryza sativa Lin.) usando dos metodologías. Agronomía Mesoamericana 5: 118-125.

Cepeda Carrión, G., y Roldán Salgueiro, J. L. (2004). Aplicando en la práctica la técnica PLS en la administración de empresas.

CIAT. (1983). Sistema de Evaluación Estándar para arroz. 2a ed. Manuel Rosero traductor y adaptador. Cali, Colombia.

Crossa, J.; Cornelius, P.L. y Yan, W. (2002). Biplots of linear-bilinear models for studying crossover genotype x environment interaction. Crop Science 42, 619-633.

Cuadra, S. A. y Marín-Fernández, V. (2018). Evaluación y selección de líneas avanzadas de arroz (Oriza sativa L.) con alto contenido de zinc en condiciones de riego y secano en Nicaragua. La Calera, 17(29), 46-50.

Díaz Solís, S. H., Morejón Rivera, R., Lucinda David, D., y Castro Álvarez, R. (2015). Evaluación morfoagronómica de cultivares tradicionales de arroz (Oryza sativa L.) colectados en fincas de productores de la provincia Pinar del Río. Cultivos Tropicales, $36(2), 131-141$. 
Díaz, S. H., Morejón, R., y Pérez, N. D. J. (2017). Comportamiento y selección de líneas avanzadas de arroz (Oryza sativa L.) obtenidas por el Programa de Mejoramiento en Los Palacios. Cultivos Tropicales, 38(1), 81-88.

González, M.R. (2001). Interacción genotipo x ambiente en guisante proteaginoso (Pisum sativum L.). Tesis de doctorado. Universidad de Valladolid, España.

González, A., de Jesús Pérez, D., Sahagún, J., Franco, O., Morales, E. J., Rubí, M. y Balbuena, A. (2010). Aplicación y comparación de métodos univariados para evaluar la estabilidad en maíces del valle Tolucaatlacomulco, México. Agronomía Costarricense.

Hernández-Rodríguez, Annia, Rives-Rodríguez, Narovis, Acebo-Guerrero, Yanelis, Diaz-de la Osa, Acela, Heydrich-Pérez, Mayra, y Divan Baldani, Vera Lucia. (2014). Potencialidades de las bacterias diazotróficas asociativas en la promoción del crecimiento vegetal y el control de Pyricularia oryzae (Sacc.) en el cultivo del arroz (Oryza sativa L.). Revista de Protección Vegetal, 29(1), 1-10.

Ibañez, M.A., Cavanagh, M.M., y Bonamico, N.C., Renzo, M.A. di, Análisis gráfico mediante biplot del comportamiento de híbridos de maíz. RIA.

INETER (Dirección General de Meteorología, Instituto Nicaragüense de Estudios Territoriales). (2018). Datos climáticos. Managua, NI.

Lozano-Ramírez, Águeda, Santacruz-Varela, Amalio, San-Vicente-García, Félix, Crossa, José, Burgueño, Juan, y Molina-Galán, José D. (2015). Modelación de la interacción genotipo x ambiente en rendimiento de híbridos de maíz blanco en ambientes múltiples. Revista fitotecnia mexicana, 38(4), 337-347.

Márquez Ruiz, C. (2017). Modelo de regresión PLS.

Morejón, R., y Díaz Solís, S. H. (2015). Selección de líneas promisorias de arroz (Oryza sativa L.) provenientes del programa de mejoramiento genético en" Los Palacios". Cultivos Tropicales, 36(4), 126-132.

Orona Castro, F., Medina Méndez, J., Tucuch Cauich, F. M., Soto Rocha, J. M., y Almeyda León, I. H. (2013). Parámetros de estabilidad en rendimiento y adaptabilidad de 25 genotipos de arroz en Campeche, México. Phyton (Buenos Aires), 82(2), $255-261$.

Pacheco A., Vargas M., Alvarado G., Rodríguez R., López M., Crossa J y Burgueño J. (2015). GEA-R (Genotype x Environment Analysis with R for Windows). Version 2.0 (2015-10-21). Copyright (C) 2015 Centro Internacional de Mejoramiento de Maíz y Trigo (CIMMYT).

Parga, T. V. M.; Zamora, V. V. M.; González, V. V. M.; García, G. S. J. y Villavicencio, G. E. E. (2005). Interacción genotipo ambiente en clones de papa bajo riego en el noreste de México. Agric. Téc. Méx. 31(1):55-64.

Pérez, L. D. J.; González, H. A.; Franco, M. O.; Rubí, A. M.; Ramírez, D. J. F.; Castañeda, V. A. y Aquino, M. J. G. (2014). Aplicación de métodos multivariados para identificar cultivares sobresalientes de haba para el estado de México, México. Rev. Mex. Cienc. Agríc. 5(2):265-278.

Samonte, S. O. P., Wilson, L. T., McClung, A. M., y Medley, J. C. (2005). Targeting cultivars onto rice growing environments using AMMI and SREG GGE biplot analyses. Crop Science, 45(6), 2414-2424.

Sanchez, A., Molina, L., y Torres, J. (2014). Métodos tradicionais e o método SREG (regressão de sítios) na seleção de genótipos em testes multiambientais. pp. 539-543. Revista da Estatística da Universidade Federal de Ouro Preto, 3(3).

Sandoval Balladares, J. D. C. (2018). Evaluación y selección de líneas avanzadas de arroz (Oryza Sativa L.) en base a características agronómicas y de rendimiento 2014-2016. Tesis de Maestría. Universidad Nacional Agraria. NI.

SAS Institute (2002). Statistical Analysis System. SAS/ETS 9 User's Guide, Volumes 1 and 2. SAS Institute Inc. Cary, NC, EUA. $2143 \mathrm{pp}$.

Téllez G. G., Rivera A.C. (2017) Producción Agricola: La cadena productiva del arroz en Nicaragua y su enfoque en la seguridad alimentaria en el ciclo 2012-2013. Universidad Nacional Autónoma de Nicaragua, Managua.

Trouchez, G.; Narváez-Rojas, L.; Chow, Z. y Corrales-Blandón, J. (2006). Fitomejoramiento participativo del arroz de secano en Nicaragua: Metodologías, resultados y lecciones aprendidas. Agronomía Mesoamericana, 2006, vol. 17, no. 3, p. $307-322$.

Wade, L. J., McLaren, C. G., Quintana, L., Harnpichitvitaya, D., Rajatasereekul, S., Sarawgi, A. K., ... y Siopongco, J. (1999). Genotype by environment interactions across diverse rainfed lowland rice environments. Field Crops Research, 64(1-2), 35-50.

Williams A., H., Pecina Q., V., Zavala G., F., Montes G.N., Gámez V., J., Arcos C., G.,... y Alcalá Salinas, L. (2010). Modelo de Finlay y Wilkinson vs. el Modelo AMMI para analizar la interacción genotipo-ambiente en sorgo. Revista fitotecnia mexicana, 33 (2), 117-123. 